\title{
Submodular sketches of single-cell RNA-seq measurements
}

\author{
Wei Yang \\ Department of Genome Sciences, \\ University of Washington
}

\author{
Jeffrey Bilmes \\ Department of Electrical and \\ Computer Engineering, University of \\ Washington \\ Paul G. Allen School of Computer \\ Science and Engineering, University \\ of Washington
}

\author{
William Stafford Noble \\ Department of Genome Sciences, \\ University of Washington \\ Paul G. Allen School of Computer \\ Science and Engineering, University \\ of Washington \\ william-noble@uw.edu
}

\begin{abstract}
Single-cell RNA-seq (scRNA-seq) datasets now routinely profile tens of thousands to millions of cells. These data are invaluable for finding important subpopulations of cells and for closely studying the mechanics of gene expression. However, as these datasets become larger, they become more difficult to analyze. Analyzing and sharing massive single-cell RNA-seq datasets can be facilitated by creating a "sketch" of the data-a selected subset of cells that accurately represent the full dataset. In this work, we use an existing benchmark to demonstrate the utility of submodular optimization in efficiently creating high quality sketches of scRNA-seq data.
\end{abstract}

\section{CCS Concepts}

\section{- Applied computing $\rightarrow$ Computational genomics.}

\section{Keywords}

single-cell expression, sketching, submodular maximization

\section{ACM Reference Format:}

Wei Yang, Jeffrey Bilmes, and William Stafford Noble. 2020. Submodular sketches of single-cell RNA-seq measurements. In Proceedings of the 11th ACM International Conference on Bioinformatics, Computational Biology and Health Informatics (BCB '20), September 21-24, 2020, Virtual Event, USA. ACM, New York, NY, USA, 6 pages. https://doi.org/10.1145/3388440.3412409

\section{Introduction}

By capturing variation of gene expression within a population of cells, single-cell RNA-seq (scRNA-seq) measurements add an additional dimension to already large gene expression datasets. As a result, scRNA-seq datasets can be massive. For example, a recent scRNA-seq analysis of 61 staged mouse embryos yielded measurements of $>2$ million cells [2]. Clearly, computing on these large datasets can be challenging for those with only modest hardware, and this problem is made worse when performing meta-analyses that aim to aggregate scRNA-seq data from multiple studies.

One common strategy to facilitate analysis of very large datasets is to identify and remove redundant examples. In scRNA-seq, this strategy can be used to select a subset of the cells in an experiment that show different patterns of gene expression. The selected subset,

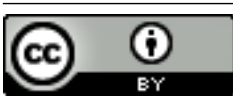

This work is licensed under a Creative Commons Attribution International 4.0 License. BCB '20, September 21-24, 2020, Virtual Event, USA

(C) 2020 Copyright held by the owner/author(s)

ACM ISBN 978-1-4503-7964-9/20/09.

https://doi.org/10.1145/3388440.3412409 sometimes referred to as a "sketch" of the full dataset, can then be analyzed using clustering or cell type assignment methods [4].

A recently described method for selecting sketches of scRNAseq data, Geosketch, is based on minimizing a particular distance function-the Hausdorff distance-between the full dataset (the "ground set") and the sketch [10]. The Geosketch algorithm can be used to produce more detailed visualizations of transcriptional activity, to reveal distinct cell types via clustering, and has been demonstrated to successfully detect a rare subpopulation of inflammatory macrophages in an umbilical cord blood cell sample [10].

Here, we propose constrained submodular maximization as a theoretically grounded and powerful framework for selecting a sketch of scRNA-seq data. Loosely speaking, submodular optimization can be considered a discrete analog of convex optimization, where the goal is to identify a set of discrete elements, rather than a realvalued vector, that optimizes an objective. We hypothesized that switching to a submodular maximization framework, with its fast algorithms and performance guarantees, would yield better (or at least as good) sketches much more quickly than Geosketch.

\section{Background}

\subsection{Submodularity}

Submodular functions are set functions that satisfy the property of diminishing returns: if we think of a function $f(S)$ as measuring the value of a sketch $S$ that is a subset of a larger set of data items $S \subseteq V$, then the submodular property means that the incremental "value" of adding a data item $s$ to a sketch $S$ decreases as the size of $S$ grows (e.g., $f(S+s)-f(S) \geq f(R+s)-f(R)$ whenever $S \subseteq R$ and $s \notin R)$. Unfortunately, searching for a sketch of maximal quality, as measured by $f(S)$, is computationally infeasible for an arbitrary set function; however, when the set function is submodular, then the quality can be approximately maximized (i.e., the quality of the identified solution is a least constant factor times optimal) in loworder polynomial time [8, 19, 21]. Moreover, the approximation ratio achieved by these optimization algorithms is provably the best achievable in polynomial time, assuming $P \neq N P$. For these reasons, submodular optimization has a long history in economics $[3,30]$, game theory $[27,28]$, combinatorial optimization $[7,18,26]$, electrical networks [20], and operations research [5]. Furthermore, submodular maximization has recently been used with great success for selecting sketches of text documents [14-16], recorded speech [17, 32, 33], image compendia [29], sets of protein sequences [13], sets of genomics assays [31], and sets of genomic loci [9]. 


\subsection{Relation to Hausdorff distance}

The Hausdorff distance that Geosketch minimizes is not submodular but can be viewed as a robust form of a commonly used submodular function. In particular, the goal of Hie et al. [10] is to minimize the Hausdorff distance between the fixed ground set (they call it $\mathcal{X}$ while we call it $V$ ) and the subset. Hence, $V$ is fixed and only $S \subseteq V$ is variable, and their goal is to perform the following optimization:

$$
\min _{S \subseteq V:|S|=k} d_{H}(V, S),
$$

where $d_{H}(T, S)=\max _{t \in T} \min _{s \in S} d(t, s)$ for arbitrary subsets $T, S \subseteq$ $V$, and where $d(t, s) \geq 0$. Note that we can replace the optimization $\min _{S \subseteq V:|S|=k} d_{H}(V, S)$ with $\min _{S \subseteq V:|S| \leq k} d_{H}(V, S)$ due to the monotone non-increasing property of $d_{H}$, i.e., for any $S \subseteq T$, $d_{H}(V, S) \geq d_{H}(V, T)$. This expression is parameterized by elementpair distances, i.e., $d(t, s)$ for $t, s \in V$. The optimization in Equation (1) is equivalent to the problem

$$
\max _{S \subseteq V:|S| \leq k} \min _{v \in V} \max _{s \in S} a(v, s)
$$

where $a(v, s)=\alpha-d(v, s) \geq 0$ is an affinity (or similarity) between $v$ and $s$ and where $\alpha$ is a positive constant. We can see this by noting that, for any set function $f$, we have $\min _{S} f(S)=-\max _{S}(-f(S))$ and so

$$
\begin{aligned}
\min _{S \subseteq V:|S|=k} \max _{t \in T} \min _{s \in S} d(t, s) & =\min _{S \subseteq V:|S|=k} \max _{t \in T}\left(-\max _{s \in S}(-d(t, s))\right) \\
& =\min _{S \subseteq V:|S|=k}\left(-\min _{t \in T} \max _{s \in S}(-d(t, s))\right) \\
& =-\max _{S \subseteq V:|S|=k} \min _{t \in T} \max _{s \in S}(-d(t, s)) .
\end{aligned}
$$

We then add a constant, replacing $-d(t, s)$ with $\alpha-d(t, s)$, setting $\alpha$ to ensure that $\alpha-d(t, s) \geq 0$, and realize that we are interested in only the argmin or argmax, so an outer minus sign is vacuous. Thus, if we define

$$
f_{r}(S)=\min _{v \in V} \max _{s \in S} a(v, s),
$$

then this objective is a minimization over a set of submodular functions, since the function $g_{v}(S)=\max _{s \in S} a(v, s)$ (assuming $a(v, s) \geq 0$ which is true if $\alpha$ above is set appropriately) is submodular in $S$ for all $v \in V$, and we have that $f_{r}(S)$ is monotone non-decreasing (meaning $f_{r}(S) \leq f_{r}(R)$ whenever $S \subseteq R$ ). A wellknown standard submodular function known as the facility location function has the form

$$
f(S)=\sum_{v \in V} \max _{s \in S} a(v, s) .
$$

When we compare $f_{r}(S)$ with $f(S)$ we see that $f(S)$ is a sum over the set of submodular functions $\left\{f_{v}(S)\right\}$ while $f_{r}(S)$ is the minimization over the same set of submodular functions. Hence, Geosketch, which maximizes $f_{r}$, maximizes the worst case over the set $\left\{f_{v}(S)\right\}$ of submodular functions. In contrast, when using the facility location function, we maximize the average case over the same set of functions. Unfortunately, the minimization over a set of submodular functions does not preserve submodularity so we cannot carry out the Geosketch maximization by using the same fast algorithms while being afforded the same mathematical guarantee [6, 23] Hence, at least until the space of submodular functions for the problem of finding sketches of scRNA-seq measurements has been fully investigated, it seems that attempting to maximize the worst case may be premature. Our results below, showing good results using a simple feature-based objective, support this suggestion.

\section{Methods}

\subsection{Data}

We downloaded four scRNA-seq datasets previously used to assess the performance of Geosketch: a 293T and Jurkat mixture dataset with 4,185 cells [35], a peripheral blood mononuclear cell (PBMC) dataset with 68,579 cells [35], a developing and adolescent central nervous system (CNS) dataset with 465,281 cells [34], and an adult mouse brain dataset with 665,858 cells [24]. For each dataset, we focused on the same rarest cell type as Geosketch: 28 293T cells ( $0.66 \%$ of the total number of cells in the dataset) in the 293T and Jurkat mixture dataset, 262 dendritic cells $(0.38 \%)$ in the PBMC dataset, 2,777 ependymal cells $(0.60 \%)$ in the mouse CNS dataset, and 1695 macrophages $(0.25 \%)$ in the adult mouse brain dataset.

\subsection{Submodular maximization}

We ran two submodular optimization toolkits-an open source Python package called "apricot" [25] and a commercial tool provided by Summary Analytics Inc. (smr.ai)-using a "feature-based" objective [11] of the form

$$
f(S)=\sum_{u=1}^{m} \sqrt{\sum_{s \in S} x_{u}(s)},
$$

where $X$ is a dataset represented as an $n \times m$ matrix with columns $x_{u}$ for feature (gene) $u, u$ is the index of a single feature in the dataset, $m$ is the number of features in the dataset, and $x_{u}(a)$ is the value of feature $u$ for example (cell) $a$. The square root function is critical, since its concavity is what provides a diminishing returns property. Note that, in general, this type of feature-based selection requires non-negative feature values (i.e., $x_{u}(s) \geq 0$ ). Both Equation (5) and Equation (4) are submodular. We chose in this work to evaluate Equation (5) rather than Equation (4) since it performs well in general and since submodular maximization using this functional form can be seen as a type of divergence minimization [1].

In the case of scRNA-seq data, the read counts are naturally non-negative. For both smr.ai and apricot we subsequently normalized the read counts for each cell to a unit vector, maintaining the non-negativity of each feature. Prior to analysis by apricot, the expression values of each gene were linearly rescaled to the range $[0,1]$. Feature-based selection was carried out by calling apricot's FeatureBasedSelection function with default parameters.

The apricot software is freely available under MIT license at https://github.com/jmschrei/apricot. The smr.ai software can be, on request, made to be used freely by academic researchers via http://smr.ai.

\subsection{Geosketch}

Geosketch was imported as a Python package obtained from http: //cb.csail.mit.edu/cb/geosketch. The "gene" dimension of each data matrix was reduced to a dimensionality of 100 PCs by randomized PCA before the sketch, as implemented in Geosketch. The matrix of 100 PCs and subset size were then passed to the gs_gap function to generate a list of indices of selected cells. Note that because the 
randomized PCA contributes to the variability of the final answer, we treat the randomized PCA as part of the Geosketch method. Hence, in contrast to the results reported in Hie et al., the error bars in Figure 1A reflect variation from both the randomized PCA and the Geosketch algorithm, rather than just the Geosketch algorithm.

\subsection{Louvain clustering}

For the Louvain clustering analysis, we followed the protocol used by Hie et al. First, the "gene" dimension of the data matrix was reduced to 100 via randomized PCA. This reduced matrix was then used to construct a nearest neighbor graph on which the Louvain community detection algorithm was applied. Louvain labels were assigned to the full dataset based on the most common label of the five nearest neighbors within the selection.

The similarity between the labels from the full dataset and from the sketch was quantified by the balanced adjusted mutual information (BAMI) score implemented by the scikit-learn Python package. This approach uses a resampled dataset, where the relative frequencies of the ground truth clusters (i.e., those derived from the full dataset) are set to uniform. The analysis was repeated with three different Louvain resolutions (0.5, 1 and 2), and the maximum BAMI score across these settings was reported.

\subsection{Immune cell analysis}

We downloaded the umbilical cord blood dataset previously used to assess the ability of Geosketch to enrich for a rare subtype of inflammatory cells. The dataset was filtered to eliminate cells with fewer than 500 genes, normalized by the total reads per cell, augmented with a pseudocount of 1 , and then natural log transformed, following the Geosketch protocol. Sketches were selected by smr.ai, apricot and Geosketch, as described in Sections 3.2-3.3. Clustering was performed on the sketches by running the Louvain community detection algorithm, as implemented in scanpy, with the default parameters (resolution of 1, 15-nearest neighbors graph), again following the Geosketch protocol.

\subsection{Timing}

To compare running times, we generated sketches of varying sizes on a single thread on a $2.70 \mathrm{GHz}$ Intel Xeon CPU E5-2680 with 250 GB of memory. Wall clock times for apricot and Geosketch were recorded using the Python "time" module. For smr.ai, we used the wall clock time reported by the software. As is common in the high-performance computing literature, we repeated each timing test 10 times and reported the minimum wall clock time, to reduce any possible effect of operating system interference.

\section{Results}

\subsection{Four benchmark datasets}

We applied apricot and smr.ai to the four benchmark datasets that were analyzed in the Geosketch paper. To evaluate the methods, we used the same performance measure employed by Hie et al., namely, the count of the number of cells of the rarest cell type that are included in a sketch of $2 \%$ of the total dataset. Here, the intuition is that a good sketch is one that undersamples common cells that presumably occupy dense regions of the transcriptional space. We observe that, in all four datasets, the sketches produced by the submodular approaches outperform Geosketch, often by a large margin (Figure 1A). Both submodular approaches use roughly the same objective function, though the commercial tool includes proprietary forms of the user-specified objective. These modifications explain the overall better performance of smr.ai relative to apricot on these evaluation tasks.

We also recapitulated a second evaluation on these same four data sets, this time quantifying the similarity of a clustering induced from the full dataset versus a clustering induced from a sketch. Here, the hypothesis is that a high quality sketch should yield a clustering that closely resembles the clustering produced from the full dataset. In this evaluation, both submodular approaches dominate the Geosketch across all four datasets and nearly all sample sizes, the only exception being the smallest apricot sketch for the PBMC dataset (Figure 1B).

\subsection{Investigation of rare inflammatory cell types in an umbilical cord dataset}

Hie et al. also demonstrate the utility of Geosketch by demonstrating its ability to discern a rare subtype of inflammatory cells within scRNA-seq data derived from an umbilical cord blood sample. We attempted to replicate this analysis by carrying out Louvain community detection on sketches produced by Geosketch, apricot, smr.ai, and uniform sampling (Figure 2A). Hie et al. report that Geosketch segregates macrophages into multiple subgroups, whereas uniform downsampling yields a single macrophage cluster. Based on expression of the CD68 (Figure 2B) and cd14 marker genes, we reach a different conclusion. In our analysis, uniform downsampling yields two macrophage clusters. The submodular approaches, on the other hand, yield three CD68 clusters (smr.ai) and two clusters (apricot).

Following Hie et al., we examined the distribution of expression values of four inflammatory marker genes (JUNB, CD74, HLA-DRA and B2M) in putative inflammatory macrophage clusters and other macrophage clusters (Figure $2 \mathrm{C}-\mathrm{F}$ ). Specifically, we computed receiver operating characteristic curves between the inflammatory macrophage clusters versus other macrophage clusters and used the area under the receiver operating characteristic curve (AUROC) as a performance measure. This analysis failed to reproduce the AUROC scores $>0.9$, as reported by Hie et al. Instead, we observe that all three sketching methods produce higher AUROC scores for all four marker genes, relative to the scores produced by uniform sampling. Thus, though the specific AUROC values differ in our analysis and that of Hie et al., the conclusion-namely, that sketching can help to reveal this particular rare cell type-is the same. Notably, this AUROC-based analysis does not discriminate between different sketching methods: all three (Geosketch, smr.ai, apricot) yield roughly comparable AUROC scores.

\subsection{Running time comparison}

Finally, we evaluated the running times of the various approaches, and concluded that the submodular approaches compare favorably to Geosketch in this respect (Figure 1C). The apricot software, which is implemented in Python using both numpy [22] and numba [12] to accelerate computation, performs comparably to Geosketch for smaller selected sets but runs more slowly as the size of the 

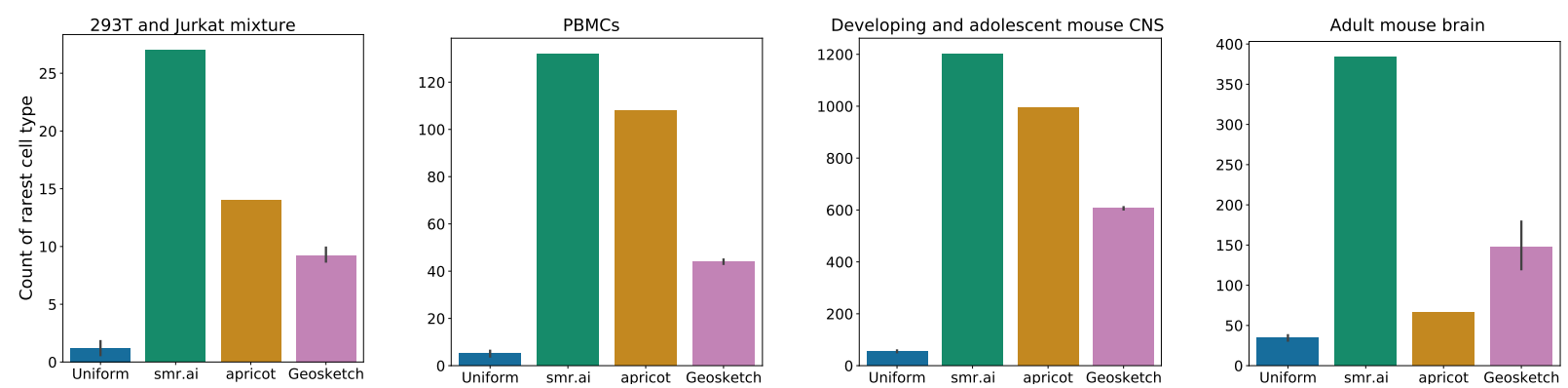

A
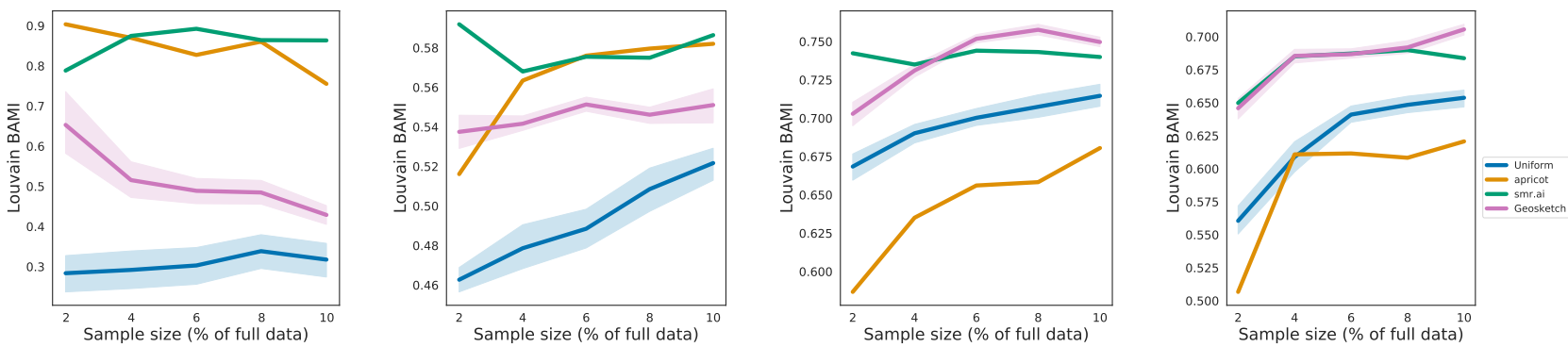

B
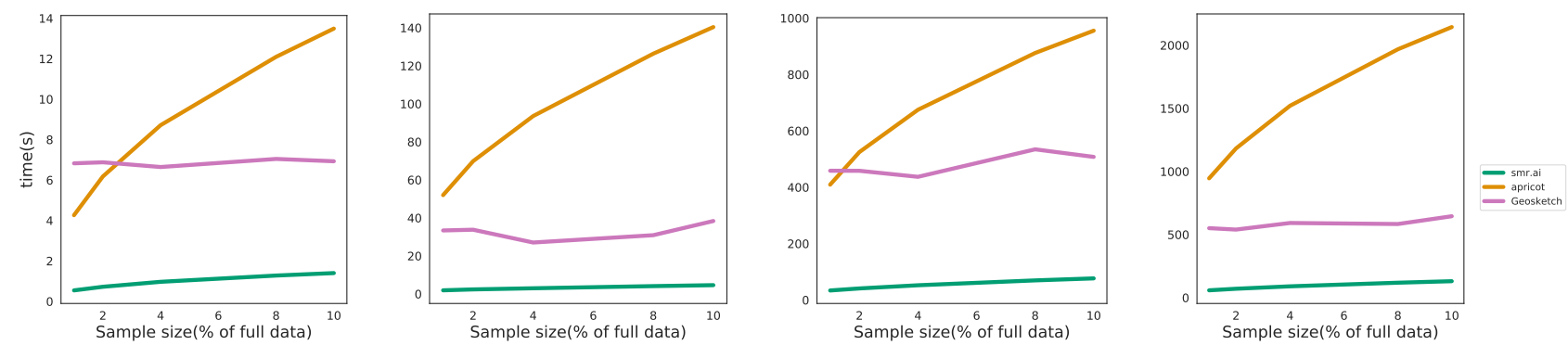

C

Figure 1: (A) Each panel plots, for a specified benchmark dataset, the number of cells of the rarest type that were selected by each of the four sketching methods. All sketches are $2 \%$ of the size of the full dataset. Error bars in the Uniform and Geosketch bars indicate 95\% confidence intervals from 10 sketches. (B) Each panel plots, for the four sketching method, the balanced adjusted mutual information between the clustering produced from a sketch of the given size ( $x$-axis) relative to a clustering produced from the full dataset. (C) Each panel compares the wall clock times required by Geosketch, apricot and smr.ai to produce sketches of varying sizes.

selected set gets larger. On the other hand, the smr.ai tool, which is implemented in $\mathrm{C}++$, is generally an order of magnitude faster than Geosketch.

\section{Discussion}

Overall, our empirical results suggest that submodular maximization provides a powerful and efficient way to summarize largescale scRNA-seq datasets. Of particular note, the results we report here have not been optimized with respect to parameter selectionindeed, they represent only the very first objective function that we tried. The space of submodular functions is vast and diverse, and particular functions can in principle be constructed to obtain particular kinds of sketches. Thus, users of such tools may wish to experiment with varying the form of the submodular objective to obtain sketches that are, for example, particularly enriched in rare cell types or that pay particular attention to capturing outliers.

An alternative to sketching scRNA-seq by selecting subsets of cells is to solve the transposed problem: find a subset of genes whose expression is representative of the entire dataset. Solving this problem is useful not only to make large data sets easier to manipulate but also for designing less expensive experimental assays. Exploring the application of constrained submodular maximization in such settings is an avenue worthy of investigation.

Acknowledgments The authors are grateful to Jacob Schreiber for helpful discussion as well as feedback on the manuscript.

Funding and competing interests This work was supported by NIH award U01 HG009395. JB holds a commercial interest in smr.ai. WY and WSN declare no competing interests. 
Uniform sampling

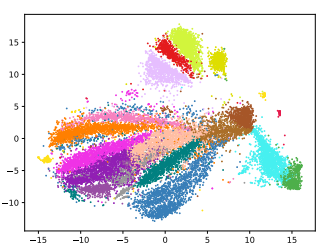

A

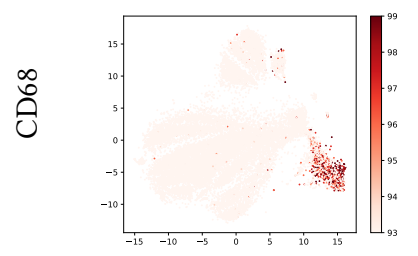

B

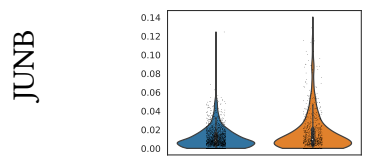

C
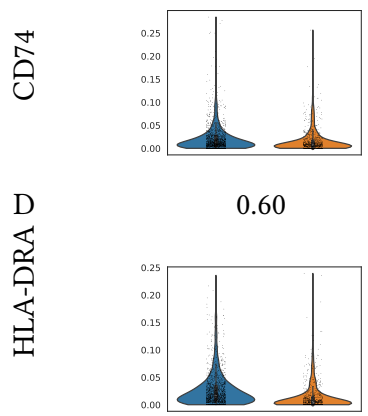

E

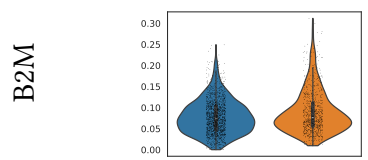

F
Geosketch

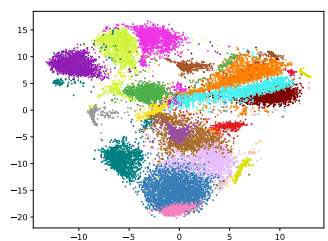

smr.ai

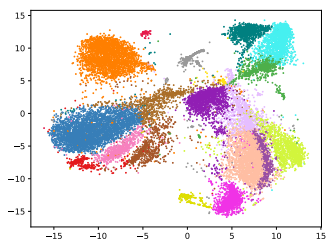

apricot

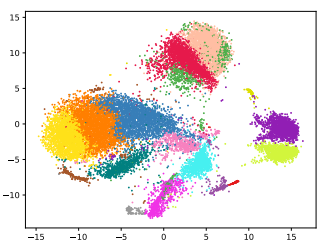

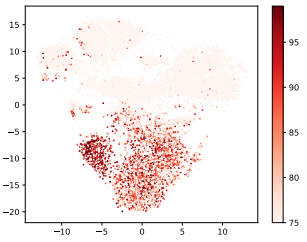

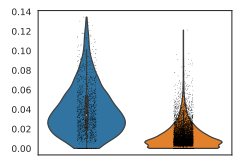

0.84

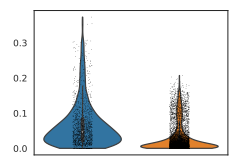

0.74

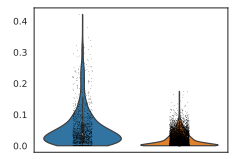

0.73

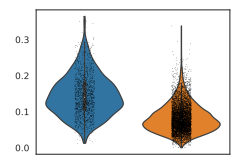

0.83
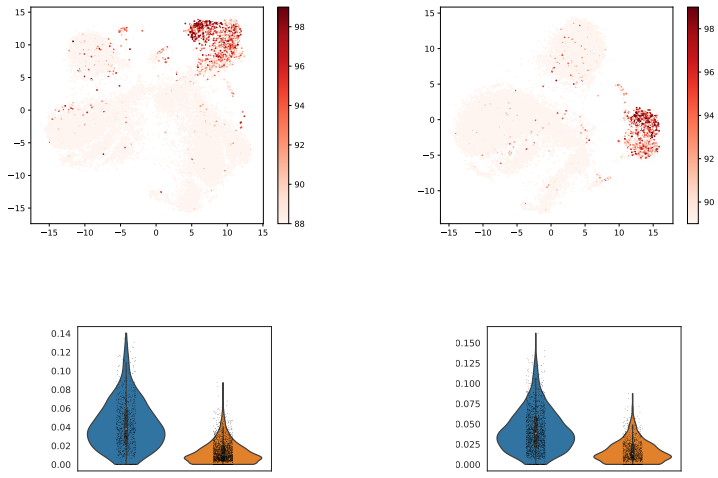

0.87

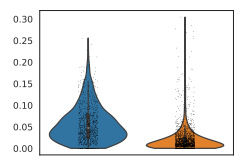

0.81

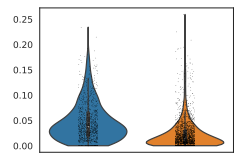

0.73

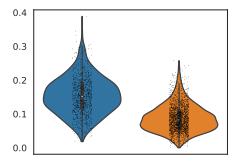

0.84

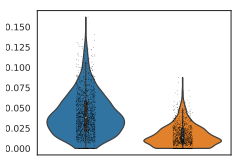

0.82

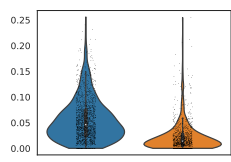

0.78

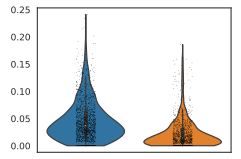

0.73

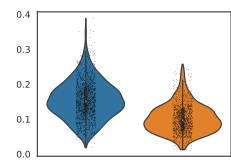

0.78

Figure 2: (A) Each plot shows a UMAP projection of one of the four datasets, with colors corresponding to the detected communities. (B) These are the same UMAP projections as panel A, but with cells colored according to CD68 expression. (C-F) Each violin plots shows the distribution of expression values for a specified marker gene (JUNB, CD74, HLA-DRA, and B2M) in a cluster enriched for inflammatory macrophages (blue) versus the remaining cluster (or clusters) of macrophages (orange). For uniform sampling, two putative macrophage clusters are compared to each other, but neither is enriched in inflammatory marker genes. The value under each plot is the corresponding AUROC.

\section{References}

[1] J. A. Bilmes and W. Bai. 2017. Deep Submodular Functions. Arxiv abs/1701.08939 (Jan 2017).

[2] J. Cao, M. Spielmann, X. Qiu, X. Huang, D. M. Ibrahim, A. J. Hill, F. Zhang, S Mundlos, L. Christiansen, F. J. Steemers, C. Trapnell, and J. Shendure. 2019. The single-cell transcriptional landscape of mammalian organogenesis. Nature 566 (2019), 496-502.

[3] M. Carter. 2001. Foundations of Mathematical Economics. The MIT Press.

[4] G. Cormode. 2017. Data sketching. Commun. ACM 60, 9 (2017), 48.

[5] G. Cornunéjols, G. L. Nemhauser, and L. A. Wolsey. 1990. The uncapacitated facility location problem. In Discrete Location Theory, P.B. Mirchandani and R.L. 
Franci (Eds.). Wiley/Interscience, New York, Chapter 3, 119-171.

[6] Andrew Cotter, Mahdi Milani Fard, Seungil You, Maya Gupta, and Jeff Bilmes. 2018. Constrained Interacting Submodular Groupings. In International Conference on Machine Learning (ICML). Stockholm, Sweden.

[7] J. Edmonds. 1970. Matroids, Submodular Functions, and Certain Polyhedra. Combinatorial Structures and Their Applications (1970), 69-87.

[8] M.L. Fisher, G.L. Nemhauser, and L.A. Wolsey. 1978. An analysis of approximations for maximizing submodular set functions-II. Polyhedral combinatorics (1978), 73-87.

[9] M. Gasperini, A. J. Hill, J. L. McFaline-Figueroa, B. Martin, S. Kim, D. Jackson, A. Leith, J. Schreiber, W. S. Noble, C. Trapnell, N. Ahituv, and J. Shendure. 2019 A genome-wide framework for mapping gene regulation via cellular genetic screens. Cell 176 (2019), 377-390. Issue 1.

[10] B. Hie, H. Cho, B. DeMeo, B. Bryson, and B. Berger. 2019. Geometric sketching compactly summarizes the single-cell transcriptomic landscape. Cell Systems 8 (2019), 483-493.

[11] K. Kirchhoff and J. Bilmes. 2014. Submodularity for data selection in machine translation. In Empirical Methods in Natural Language Processing (EMNLP).

[12] S. K. Lam, A. Pitrou, and S. Seibert. 2015. Numba: A LLVM-based Python JIT Compiler. In Proceedings of the Second Workshop on the LLVM Compiler Infrastructure in HPC (LLVM '15). ACM, New York, NY, USA, 7:1-7:6.

[13] M. W. Libbrecht, J. A. Bilmes, and W. S. Noble. 2018. Choosing non-redundant representative subsets of protein sequence data sets using submodular optimization. Proteins 86, 4 (2018), 454-466.

[14] G. Lin, M. K. Chawla, K. Olson, C. A. Barnes, J. F. Guzowski, C. Bjornsson, W. Shain, and B. Roysam. 2007. A multi-model approach to simultaneous segmentation and classification of heteregenous populations of cell nuclei in 3D confocal microscope images. Cytometry A. 71, 9 (2007), 724-736.

[15] H. Lin and J. Bilmes. 2011. A class of submodular functions for document summarization. In Proceedings of the 49th Annual Meeting of the Association for Computational Linguistics: Human Language Technologies-Volume 1. Association for Computational Linguistics, 510-520.

[16] H. Lin and J. Bilmes. 2012. Learning Mixtures of Submodular Shells with Application to Document Summarization. In Uncertainty in Artificial Intelligence (UAI) AUAI, Catalina Island, USA, 479-490.

[17] Y. Liu, K. Wei, K. Kirchhoff, Y. Song, and J. Bilmes. 2013. Submodular feature selection for high-dimensional acoustic score spaces. In Acoustics, Speech and Signal Processing (ICASSP), 2013 IEEE International Conference on. IEEE, 71847188

[18] L. Lovász. 1983. Submodular functions and convexity. In Mathematical Programming - The State of the Art, M. Grotchel A. Bachem and B. Korte (Eds.) Springer-Verlag, Bonn, 235-257.

[19] M. Minoux. 1978. Accelerated greedy algorithms for maximizing submodular set functions. Optimization Techniques (1978), 234-243.

[20] H. Narayanan. 1997. Submodular functions and electrical networks. Annals of Discrete Mathematics 54 (1997).

[21] G. L. Nemhauser, L. A. Wolsey, and M. L. Fisher. 1978. An analysis of approximations for maximizing submodular set functions. Mathematical Programming 14, 1 (1978), 265-294.

[22] T. E. Oliphant. 2006. Guide to NumPy. CreateSpace Independent Publishing Platform, North Charleston, SC.

[23] T. Powers, J. Bilmes, D. W. Krout, and L. Atlas. 2016. Constrained Robust Submodular Sensor Selection with Applications to Multistatic Sonar Arrays. In 19th International Conference on Information Fusion. IEEE, Heidelberg, Germany.

[24] A. Saunders, E.Z. Macosko, A. Wysoker, M. Goldman, F.M. Krienen, H. de Rivera, E. Bien, M. Baum, L. Bortolin, S. Wang, A. Goeva, J. Nemesh, N. Kamitaki, S. Brumbaugh, D. Kulp, and S. A. McCarroll. 2018. Molecular diversity and specializations among the cells of the adult mouse brain. Cell 174 (2018), 999-1014.

[25] J. M. Schreiber, J. Bilmes, and W. S. Noble. 2019. apricot: Submodular selection for data summarization in Python. arXiv (2019). https://arxiv.org/abs/1906.03543.

[26] A. Schrijver. 2004. Combinatorial Optimization. Springer.

[27] L. S. Shapley. 1971. Cores of convex games. International fournal of Game Theory 1, 1 (1971), 11-26.

[28] D. M. Topkis. 1998. Supermodularity and complementarity. Princeton University Press.

[29] S. Tschiatschek, R. K. Iyer, H. Wei, and J. A. Bilmes. 2014. Learning mixtures of submodular functions for image collection summarization. In Advances in Neural Information Processing Systems. 1413-1421.

[30] X. Vives. 2001. Oligopoly pricing: Old ideas and new tools. The MIT Press.

[31] K. Wei, M. W. Libbrecht, J. A. Bilmes, and W. S. Noble. 2016. Choosing panels of genomics assays using submodular optimization. Genome Biology 17, 1 (2016), 229

[32] K. Wei, Y. Liu, K. Kirchhoff, C. Bartels, and J. Bilmes. 2014. Submodular subset selection for large-scale speech training data. In Acoustics, Speech and Signal Processing (ICASSP), 2014 IEEE International Conference on. IEEE, 3311-3315.

[33] K. Wei, Y. Liu, K. Kirchhoff, and J. Bilmes. 2013. Using Document Summarization Techniques for Speech Data Subset Selection.. In HLT-NAACL. 721-726.
[34] A. Zeisel, H. Hochgerner, P. Lönnerberg, A. Johnsson, F. Memic, J. van der Zwan, M. Häring, E. Braun, L.E. Borm, G. La Manno, S. Codeluppi, A. Furlan, K. Lee, N. Skene, K. D. Harris, J. Hjerling-Leffler, E. Arenas, P. Ernfors, U. Marklund, and S. Linnarsson. 2018. Molecular Architecture of the Mouse Nervous System. Cell 174 (2018), 999-1014.

[35] G. X. Y. Zheng, J. M. Terry, P. Belgrader, P. Ryvkin, Z. W. Bent, R. Wilson, S. B. Ziraldo, T. D. Wheeler, G. P. McDermott, J. Zhu, M. T. Gregory, J. Shuga, L. Montesclaros, J. G. Underwood, D. A. Masquelier, S. Y. Nishimura, M. SchnallLevin, P. W. Wyatt, C. M. Hindson, R. Bharadwaj, A. Wong, K. D. Ness, L. W. Beppu, H. J. Deeg, C. McFarland, K. R. Loeb, W. J. Valente, N. G. Ericson, E. A. Stevens, J. P. Radich, T. S. Mikkelsen, B. J. Hindson, and J. H. Biela. 2017. Massively parallel digital transcriptional profiling of single cells. Nature Communications 8 (2017), 14049 